\title{
Preferential void formation at crystallographically ordered grain boundaries in nanotwinned copper thin films
}

\author{
Thomas LaGrange ${ }^{1}$, Kazuto Arakawa ${ }^{2,3}$, Hidehiro Yasuda ${ }^{4}$, and Mukul Kumar ${ }^{1}$ \\ ${ }^{1}$ Lawrence Livermore National Laboratory, 7000 East Avenue, L-342, Livermore, CA 94550, USA \\ ${ }^{2}$ Department of Materials Science, Faculty of Science and Engineering, Shimane University, 1060 \\ Nishikawatsu, Matsue 690-8504, Japan \\ ${ }^{3}$ CREST, JST, 7, Gobancho, Chiyoda-ku, Tokyo 102-0076, Japan \\ ${ }^{4}$ Research Center for Ultra-High Voltage Electron Microscopy, Osaka University, 7-1 Mihogaoka, Ibaraki, \\ Osaka 567-0047, Japan
}

\begin{abstract}
:
Nanocrystalline materials are expected to have improved radiation resistance as the high density of grain boundary area is thought to act as an effective sink for radiation-induced defects. However, continued absorption of defects can alter the structure of grain boundaries and/or enhance their mobility, eventually leading to microstructural degradation in the form of grain coarsening, thus negating their initial radiation tolerance. Hence, an ideal microstructure might be one with a mix of boundaries that are effective sinks and limit grain coarsening. We show through in situ electron irradiation experiments, however, that this is an insufficient condition. Our observations indicate that even a high density of low energy coherent twin boundaries, supposedly stabilizing the microstructure against grain coarsening, can be a detriment in that it biases the mobility of vacancies accumulating during irradiation thereby resulting in preferential void nucleation near twin boundaries. These observations highlight the fact that radiation induced grain boundary migration depends greatly on the topology of the grain boundary network and that the migration of high-angle grain boundaries can be hindered when coordinated at triple junctions composed of at least two low-energy boundaries, e.g., coincidence site lattice boundaries.
\end{abstract}




\section{Introduction:}

Most structural materials used in nuclear reactors are prone to radiation damage that degrade their mechanical properties and limit their service life. High-energy particle irradiation produces defects in these materials that are mobile at high temperatures and are influenced by stress fields associated with pre-existing extended defects. After an initial relaxation phase, the defects, that do not recombine, aggregate to produce defect clusters or diffuse rapidly to interfaces and other defects, inducing detrimental microstructural evolution [1]. For example, interstitials, being more mobile than the vacancies, are quickly absorbed by nearby dislocations, inducing creep by dislocation climb and dislocation multiplication that result in work hardening and embrittlement. Likewise, a small excess of remnant vacancies can agglomerate, leading to the formation of voids that cause swelling [1-6], an increase in residual stresses, microcrack formation, and the eventual failure of the material. The longterm stability of a microstructure under irradiation depends on its neutrality toward defect absorption. Even a small imbalance in defect mobility and absorption at sinks can eventually lead to a dramatic degradation of strength and structural integrity. This could be particularly true under high radiation doses and dose rates, where the balance between the rate of defect production and absorption may overwhelm even a highly efficient defect sink $[1,7]$. Development of materials with a high density of sites that can act as sinks for the point defects produced by high-energy particles would thus be an enabling technology for reliable and clean nuclear energy.

Polycrystals with nano-sized grains have a high interfacial areal density and have been considered to possess an ideal microstructure for radiation resilience [8]. Extensive research on the void nucleation and swelling produced by high energy electron and fission neutron irradiation has shown that in materials with submicron grain sizes, void formation is greatly reduced [7, 9-12]. The main supposition of these studies is that grain boundaries (GB) are efficient sinks for vacancies and that as the average grain size decreases below $1 \mu \mathrm{m}$ vacancy concentrations dramatically diminish thereby reducing the 
tendency to form and grow voids. (The assumption that all grain boundaries behave alike regardless of their crystallographic identity is implicit in these studies.) Thus the use of nanocrystalline materials should be an adept solution to reduce irradiation induced swelling. However, at elevated temperatures the grain boundary area per unit volume in most nanocrystalline materials tends to decrease with time, potentially negating its initial advantages for radiation resilience. Remarkably, with our current knowledge of the basic physics of coarsening in high dose radiation environments, it is difficult to predict whether a given nanostructured material will have adequate radiation resistance. Moreover, nanocrystalline structures have a distinct topological character that is different from conventional micron-sized polycrystalline microstructures $[13,14]$, which may significantly influence the defect dynamics during the cascade relaxation phase. And, like the case of grain coarsening, the topological characteristics of the grain boundary network (GBN) may well be an important factor in the radiation resistance of nanostructured materials.

Here, we examine the effect of GBN topology on radiation induced grain boundary migration (RIGBM) and on void formation using nanotwinned copper (nt- $\mathrm{Cu}$ ) foils. The nt-Cu samples are composed of regions with a high density of coherent twin boundaries (CTB) and have a moderate fraction of random high angle grain boundaries (HAGB). With such topologically distinct microstructures, we are able to contrast different GB types and gain better understanding of their influence on the radiation induced coarsening and void formation (See Fig. 1 A and B). Being low energy, a high density of CTBs and triple junctions (TJ) composed of low angle boundaries (LAGB, angular misorientation $<15^{\circ}$ ) and CTBs can limit thermal coarsening in nanocrystalline materials $[15,16]$, but the sink efficiency of such microstructures is poor. Conversely, a mixture of random HAGB and CTBs is prone to destabilization if the majority of the TJs have only one low energy component (out of three), with incoherent twin boundary (ITB) segments readily nucleating and causing the hitherto low mobility interfaces to migrate at a faster rate [13] . This 
is unlike the case of conventional polycrystalline microstructures where a high density of CTBs and ITBs in a well-organized topological network substantially reduced the coarsening rate [17].

In an effort to elucidate the optimal microstructure that combines resistance to both microstructural coarsening and radiation damage we use high-energy electron irradiation in situ in a $2 \mathrm{MeV}$ transmission electron microscope (TEM). This allows us to probe the influence of GB character and network topology on the point defect annihilation and agglomeration processes attendant in nanocrystalline materials with a high density of CTBs (to provide coarsening resistance) and random HAGB (for radiation sink efficiency). By irradiating with high-energy electrons, that produces Frenkel defects (vacancy-interstitial pairs), we can isolate and observe the microstructural evolution solely associated with point defect agglomeration dynamics. We also contrast the microstructural evolution under ambient and high temperature electron radiation to evaluate the influence of GB character on damage accumulation behavior that leads to void formation. Through in situ observations of the microstructural evolution and the differences under irradiation of various grain boundary types, we are able to formulate a better correlation between grain boundary character, radiation sink efficiency and radiation defect dynamics in nanotwinned metals.

\section{Methods:}

\section{nt-Cu sample preparation}

High-purity (99.999\%) nt-Cu samples used in these studies were fabricated via DC magnetron sputtering implementing a "multilayer" process method that yields thick ( $>25 \mu \mathrm{m})$, uniform foils with a nanotwinned, columnar structure. The growth twin density in nt-Cu samples is governed by the deposition rate [18]. Depositing the foils at rates of $\sim 2.5 \mathrm{~nm} \mathrm{~s}^{-1}$ resulted in high twin area densities per volume of $>10^{7} \mathrm{~m}^{-1}$. Cross-sectional TEM samples were prepared by focused-ion-beam (FIB) method (FEI Nova 600 Dual-Beam FIB, Oregon, USA) and FIB sections were taken from the as-deposited nt-Cu films, 
which have a high density of CTBs (see orientation and bright-field images in Fig. 1). The FIB sectioning creates a thin damage layer due to the high-energy ion bombardment. To remove this amorphous damage layer, the samples were subjected to a final polishing step in a Gatan precision ion polishing sytem (PIPS) using a modified low energy Ar gun at $500 \mathrm{eV}$.

\section{Electron irradiation conditions}

In-situ electron irradiation experiments on the nt-Cu foils were conducted on the Hitachi H-3000 ultra high voltage (UHV) transmission electron microscope (TEM) located at the Research Center for High Voltage Electron Microscopy in Osaka University. The TEM was operated at a voltage of $2 \mathrm{MeV}$ and the electron beam was focused to a spot $1 \mu \mathrm{m}$ in diameter, producing electron dose rates of $2.4 \times 10^{24}$ electrons $\mathrm{m}^{-2} \mathrm{~s}^{-1}$ in the region of interest on the specimen. Images were taken sequentially as a function of irradiation time and during the imaging itself the electron beam was defocused to give effective electron doses of $\sim 1-2 \times 10^{23}$ electrons $\mathrm{m}^{-2} \mathrm{~s}^{-1}$. Electron irradiation during image acquisition itself, though minimal, was also included in these dose calculations. In-situ observations of microstructural evolution during electron irradiation were made at ambient temperatures and at higher temperatures around 573K. These conditions were chosen based on the prior research by Niwara et al. [19] who observed significant void formation in copper at these temperatures under electron irradiation [19]. Defect evolution and void formation were tracked as a function of irradiation time and calculated dose. The position of individual GBs was also tracked during irradiation to compare the influence of GB type on their observed radiation induced migration.

\section{Crystallographic orientation acquisition and analysis}

Automated crystallographic orientation mapping (ACOM) data of the nt-Cu TEM foils and irradiated materials were acquired using the NanoMEGAS ASTAR system attached to a field-emission FEI CM300 transmission electron microscope (TEM). The ASTAR system collects and indexes nanodiffraction patterns over a total scan area of a few micrometers, and thus provides statistically relevant information 
of the grain orientation, texture and GB character (i.e., GBs and TBs in general) [20]. As the thickness of TEM samples is on the order of $\sim 100-150 \mathrm{~nm}$, the dynamical (multiple scattering) diffraction effects influence the intensity of the diffraction spots and complicate the determination of the local crystal orientation. By precessing the electron beam about the TEM optical axis, which effectively wobbles the Ewald's sphere about the microscope axis, more diffraction spots are present in the diffraction patterns and quasi-kinematical diffraction intensities are obtained, greatly assisting orientation determination and increasing data reliability. The ACOM data displayed in Fig. $1 \mathrm{~b}$ were collected with a precession angle of $0.5^{\circ}$, a probe size of $6 \mathrm{~nm}$, and a scan step size of $4 \mathrm{~nm}$, which provides oversampling and increased reliability. The orientation data shown in Fig. 4 was acquired with a $1 \mathrm{~nm}$ probe size, scan step of $0.88 \mathrm{~nm}$ and a precession angle of $0.5^{\circ}$. The technique is not entirely free of artifacts; in particular, ambiguities in orientation indexing arise for regions aligned close to a high symmetry axis or when two crystals (grains) significantly overlap. These regions are infrequently encountered and are easily identified and eliminated from the statistics by partitioning the data. For example, unreliable data (pixels) below a confidence index of 0.2 were excluded and colored black in the inverse pole figure orientation maps (IPFOM). A confidence index of 1 means 100\% confidence in the orientation determination and the average confidence index of the maps in this study was $\sim 0.4$. The data was further analyzed using the neighbor orientation correlation algorithm available in $\mathrm{OIM}^{\odot} 6.5$ software by TSL-EDAX ${ }^{\circledast}$. The GB character maps were generated to show the change in the GBN of nt-Cu induced by irradiation. The boundaries were colored coded; high angle boundaries with a misorientation $>15^{\circ}$ are colored black, low angle boundaries $<15^{\circ}$ are colored yellow, and $\Sigma 3$ and $\Sigma 9 \mathrm{CSL}$ boundaries are colored red and blue, respectively.

\section{Results:}

Topology of the nt-Cu films used in irradiation studies 
The sputter deposited nt-Cu foils used in this study exhibit a strong <111>-fiber texture and have a GBN consisting of high-angle random columnar boundaries that are internally twinned and have a small fraction of LAGB interspersed with crystallographically special boundaries (as described by the coincidence site lattice (CSL model [21]). Figure 1 shows TEM micrographs and crystallographic orientation maps of the nt-Cu foil used in the electron irradiation experiments. Many of the columnar grains have a ladder-like structure of $\{111\} \Sigma 3$ CTBs aligned perpendicular to the film growth direction (Figs. 1 A), B) and D)) resulting from high deposition rates and growth conditions occurring in the multilayer sputtering technique $[13,18]$. Most columnar boundaries are random HAGB (black), but some columnar domains are bounded by LAGB, in areas with numerous triple junctions composed of $\Sigma 1$ (yellow) and $\Sigma 3$ (red) CSL boundaries (Fig. 1 D). The misorientation angle distributions of nt-Cu samples are comparable to the uncorrelated, untextured material (McKenzie distribution) with the exception of a slight enhancement of LAGBs ( $<15^{\circ}$ misorientation) and much higher fraction of $60^{\circ} \Sigma 3 \mathrm{CSL}$ boundaries. The unique microstructural topology and mixture of different grain boundary types occurring in these nt-Cu foils allows us to investigate and test different theories on how GB character influences point defect dynamics associated with irradiation damage.

\section{Ambient temperature $\mathrm{MeV}$ electron irradiation experiments}

The dashed-lined square in Fig. 1 B) indicates a region containing a high density of CTBs, a moderate fraction of random HAGBs, and a few LAGBs that was exposed to a $2 \mathrm{MeV}$ electron beam with an effective dose rate of $2.4 \times 10^{24} \mathrm{e}^{-} \mathrm{m}^{-2} \mathrm{~s}^{-1}$. The initial microstructure, prior to electron irradiation, has a discernible density of stacking fault tetrahedra (SFT) and dislocation loops formed during the FIB milling and preparation of the TEM cross-sectional sample (Fig. 2A)). After 60 mins of irradiation to a total dose of $8.7 \times 10^{27} \mathrm{e}^{-} \mathrm{m}^{-2}$ at room temperature, there was some interaction of the Frenkel defects formed by the high-energy electron irradiation with the pre-existing defects, leading to an increase in the number of SFTs and dislocation loops in region (See Fig. 2B). Slight changes in the diffraction contrast of the TEM 
images were observed after irradiation and attributed to the formation of new defects and the annihilation of pre-existing defects resulting in a change of local residual stresses. Changes in tilt of the foil relative to the electron beam during irradiation were negligible, indicating that the evolution of the defect population was changing the local stress state only minutely. Grain boundary migration under irradiation was the prominent observation; Fig. $2 \mathrm{C}$ shows traces of the HAGBs and LAGBs that evolved in the microstructure. Even with the caveat that the TEM foil surfaces may impede the motion of inclined grain boundaries, these observations lend credence to the fact that low-energy, and more stable, CSL type $\Sigma 3$ and $\Sigma 9$ boundaries are less mobile than random HAGBs and even LAGBs. This may also provide indirect evidence in support of our claims that HAGBs are more efficient sinks and that the absorption of defects may enhance GB mobility, which promotes coarsening.

\section{In-situ MeV electron irradiation at $573 \mathrm{~K}$}

At elevated temperatures, the dynamics of defect formation and their evolution are quite different. Similar to the ambient temperature irradiation experiments, the irradiation region was composed of columnar grains with a high density of CTBs and columnar boundaries having a mixture of random HAGBs and LAGBs and also contained numerous defects produced by the FIB preparation (Fig. 3A)). The sample was held at $573 \mathrm{~K}$ for 30 mins prior to irradiation until sample drift was minimal, and no distinct changes in the microstructure or grain boundary motion were observed after this short anneal. However, upon irradiation for 6 mins to a dose of $8.6 \times 10^{26} \mathrm{e}^{-} \mathrm{m}^{-2}$, numerous voids appeared having the highest densities around the CTBs (Fig. 3B)). A comparison of Fig. 3 A) and B) suggested that the small pre-existing defects created by FIB sectioning in the interior of the columnar grains disappear during the short anneal and upon further irradiation. Presumably, the combination of enhanced defect mobility and associated dynamics operating at elevated temperature act to remove small defect clusters by annihilation with Frenkel defects. Under further irradiation at $573 \mathrm{~K}$ for a period of 44 mins, the voids coarsen, coalesce and facet until they impinge on grain boundaries and/or the free surfaces of the TEM 
foils (See Fig. 3C)). Once formed, the voids may act as the dominant radiation sink, growing due to the flux of vacancies that would otherwise aggregate and produce SFTs as observed in the ambient electron irradiation experiments.

Grain boundary migration was also evaluated, and as was observed for ambient temperature electron irradiation, significant motion was observed only for specific grain boundaries. Fig. 3 D) shows traces of a set of grain boundaries near the center of the irradiated zone. In particular, the low-angle grain boundary $\left(7^{\circ}\right.$ misorientation angle about the $[\overline{20} 7 \overline{10}]$ rotation axis) located in the center of the electron irradiated zone migrated a distance of about $100 \mathrm{~nm}$ (Fig.3 D)). Other non-special boundaries were observed to change slightly after irradiation, though the CSL type boundaries were not observed to migrate. This suggests that even at high temperatures, where significant boundary migration is expected in pure $\mathrm{Cu}$, the low-energy, crystallographically ordered boundaries are less mobile.

Post mortem crystallographic analysis of the irradiated zone revealed that the highest density of voids formed around $\Sigma 3$ CSL type boundaries, particularly CTBs, whereas very few voids were observed in the vicinity of random HAGBs (See Fig. 4). Void formation is typically related to biased point defect diffusion kinetics. The coordinated structure and low free volume of $\Sigma 3$ CSL type boundaries influence defect evolution such that a local excess of vacancies accumulate, causing voids to nucleate and grow. Though not fully understanding the radiation mechanism involved, Norris also observed the preferential void formation around CTBs, noting that very few voids formed around incoherent twin boundaries [5, 22]. Likewise, we observe few voids in the vicinity of incoherent twins and RHAGB and LAGBs that are adequate sinks for both vacancies and interstitials. The fact that both random HAGBs and LAGBs were observed to move during irradiation may be indirect evidence for biased point defect mobility and absorption; the absorption of point defects may enhance their mobility under irradiation. 


\section{Discussion:}

\section{Radiation induced grain boundary migration (RIGBM)}

Previous theoretical [23] and experimental [24, 25] studies have attributed RIGBM to the diffusion of excess point defects and defect clusters to nearby GBs, with a resulting change in structure because of absorption. For RIGBM to occur two criteria are required to be met; (1) the GB must be able to accommodate point defects, i.e., have sufficient free volume, and (2) the atomic rearrangement and GB motion lowers interfacial energy, i.e., restructuring of the interface must provide the necessary driving force. Boundaries saturated with interstitials can emit these interstitials that in turn can readily diffuse and annihilate with vacancies in the interior of the grains $[8,26,27]$. Being enriched with interstitials can also lower the diffusion barrier for vacancies to migrate and recombine with interstitials in the GB, promoting its restructuring. These criteria appear to hold for the current studies with only the HAGB, which have high energy and sufficient free volume, appearing to migrate, as shown in Figs. 2 C) and 3 D). However, the low energy CSL type boundaries do not move, which suggests that since a lack of excess free volume limits accommodation and absorption of defects, thus limiting RIGM. We could not assess the change in the GB structure as the crystallographic orientation analysis only provides 3 of the 5 degrees of freedom that are related to the boundary structure. A change in the angular misorientation of the GB was not observed after irradiation, though if the boundary were restructured due to absorption of defects, a change in boundary plane inclination could be expected as an easier mechanism for boundaries to reduce their energy [28]. Regardless, trends in the observations suggest that boundaries with lower free volume and lower interfacial energy are less likely to migrate under irradiation.

A previous study ([24]) has also suggested that GB migration only occurs when there is a difference in the defect density between adjacent grains driving the boundary to migrate toward regions of lower defect density. This implies that the grain with fewer defects has a better sink efficiency and that the 
migration of the grain boundaries can only occur when an asymmetry in the defect population emerges in the initial stages of irradiation before defect saturation occurs [24]. The sink efficiency of a grain depends on the character of the neighboring GBs, and thus RIGBM depends to a greater extent on the topological aspects of the GBN that give rise to local asymmetries in the defect distributions. In addition, if the topological connectivity of GBs has a low-energy configuration that resists thermal coarsening, e.g., GBN having a high fraction of the triple or quadruple junctions composed of 2 or more CSL type boundaries, it is unlikely that RIGBM would occur in such networks. This is supported by the observations shown in Fig. 2 C). The high-angle random grain boundary with a misorientation of $54^{\circ}$ about the $[\overline{13} 9 \overline{5}$ ] axis (close to a $\Sigma 81$ CSL type) is pinned by two TJs composed of 2 CSL boundaries ( $\Sigma 3$ and $\Sigma 27 a$ ). It migrates, under irradiation, to a lesser degree than the boundary with a misorientation of $52^{\circ}$ about $[\overline{6} \overline{9} 11]$ axis, which is connected to TJs coordinated with only 1 CSL boundary. Both GBs are HAGB, but differences in the surrounding network of GBs promote one boundary to move more than the other. Similar conditions may also explain the significant migration of the LAGB $\left(7^{\circ}\right.$ misorientation) shown in Fig. 3 D), which was also not anchored by TJs having a low-energy configuration. Thus, the conditions for RIGBM may not depend solely on the energy of an individual boundary and its available free volume to absorb radiation defects, but also on its connectivity to surrounding boundaries of the GB network.

\section{Preferential void formation at high temperatures}

Nanocrystalline materials, having a higher specific volume of interfacial area, are thought to exhibit less swelling [29]. Singh et al. formulated a defect depletion model (DDM) to explain the decrease in void swelling with decreasing grain size based on the assumption that GBs act as unbiased sinks for radiation induced point defects and would never exhibit saturation [11]. This assumption may break down for highly ordered, low energy grain boundaries, e.g., coherent twin boundaries (CTBs) that lack the free volume to accommodate interstitials and vacancies. Thus, CTBs may be inefficient radiation sinks that 
saturate readily and that the assumptions and observed trends in the DDM model of Singh et al. may not hold for nanotwinned metals. Based on the observations presented here, we speculate that preferential void formation around CTBs results from their inability to act as sinks for radiation-induced vacancies that accumulate rapidly under the high electron irradiation dose rates giving rise to void nucleation.

As we mentioned earlier, interpretations and models of radiation damage and defect evolution generally assume that grain boundaries are unsaturable sinks having similar sink strengths [11], and are generally isotropic, though many studies on the variation in size and distribution of void denuded zones (VDZ) have shown that grain boundaries indeed possess different sink efficiencies [7, 19, 30, 31]. For example, coherent twin boundaries (CTBs), such as the $\Sigma 3<110>\{111\}$ tilt boundary common in f.c.c. metals, have the lowest free energy amongst grain boundaries [32], and in the studies by Han el al [30], no discernable VDZ were observed in the vicinity of CTBs. The ability of a grain boundary to absorb defects depends on its crystallographic structure, i.e., on both the misorientation and boundary plane inclination angle [30]. Low crystallographic order, high-energy GBs, with high free volume, have many more degrees of freedom that allows easy incorporation of point defects and defect clusters into the underlying boundary structure without triggering a critical transition. Conversely, perfect CTBs have a high degree of crystallographic order suggesting that they are not good sinks for vacancies and likely develop a VDZ in their vicinity. Unlike CTBs, incoherent twin boundaries (ITBs), e.g., $\Sigma 3<110>\{211\}$ tilt boundary, exhibit a VDZ owing to the relatively open structure of the $\{112\}$ boundary plane, but with a width much smaller than those observed around HAGB [30]. Though the crystallographic structure of a GB may influence the sink behavior, experimental studies and atomistic models conclude that it does not impede the formation of vacancies and vacancy clusters [33].

The mechanism by which vacancies accumulate and voids form around CTBs results from the biased motion of point defects arising from the topological constraints of the nt-Cu microstructure. At the 
elevated temperature used in this study, both vacancies and interstitials are mobile, though the characteristic diffusion distance for vacancies and interstitials are quite different. Being more mobile, the interstitials that do not recombine with vacancies have a higher probability to diffuse and annihilate at the free surfaces of the TEM foil or nearby GBs that can accommodate them. Recent molecular dynamic (MD) simulations of radiation defect dynamics near GBs showed that interstitials can easily diffuse across low-energy, coordinated CSL boundaries into adjacent grains via an absorption-emission mechanism [8]. Thus, we can conclude that CTBs do not impede the diffusion of interstitials. Furthermore, MD studies of Mendelev et al. on interstitial cluster dynamics revealed that about 9 to 12 interstitials in a cluster could be accommodated in CTBs, organizing into platelet arrays that act as effective sinks for interstitials [31]. These simulations suggest that CTBs can be permeable boundaries for interstitials. Conversely, CTBs are not effective sinks for vacancies, and being less mobile an excess of vacancies could accumulate, in the process producing voids if a more efficient sink within the characteristic diffusion distance at these temperatures is not to be found. As mentioned previously, vacancies can be accommodated into high energy boundaries, e.g., HAGB, where they either recombine with interstitials or occupy sites that reorganize the structure in the boundary to reduce its energy. The observed migration of both HAGB and LAGB is indirect proof that large amounts of point defects were incorporated into these boundaries causing them to move. On the other hand, there is a large energy cost for incorporating a vacancy into a low-energy CTB, and thus CTBs are not efficient sinks for vacancies. In a nt-Cu sample, where the average spacing between the twin boundaries in each column is on a scale less than the TEM foil thickness, vacancies produced between two CTBs that do not recombine with interstitials are unlikely to migrate as there are no effective sinks within striking distance and thus get pinned between the CTBs. The interstitials, however, being more mobile and able to migrate through CTB, can annihilate at effective sinks located at distances much larger than the twin 
width. Thus due to this biased mobility, vacancies tend to accumulate near CTBs giving rise to the observed higher densities of large voids in the vicinity of CTBs.

\section{Conclusions:}

Using in situ electron irradiation under high voltage conditions and post mortem TEM-based orientation mapping, we have studied the irradiation induced damage and microstructural evolution in Cu thin films that comprise a high density of coherent twin boundaries distributed in a "ladder-like" morphology within columnar grains that are bounded by high angle grain boundaries. The in-situ observations were made at two different temperatures, room temperature and $573 \mathrm{~K}$, a temperature known to stimulate void nucleation and growth [19], and the main findings are summarized below:

- Radiation induced grain boundary migration (RIGM) of several high-angle random and lowangle grain boundaries within the irradiated zone was observed at both temperatures. However, low-energy CSL type boundaries were not observed to migrate even at elevated temperatures.

- RIGM was not observed for grain boundaries connected to junctions that contain one or more $\Sigma 3$ boundaries. These boundaries stabilize the microstructure and suppress radiation induced coarsening.

- In the experiments conducted at $573 \mathrm{~K}$, voids were predominantly observed to nucleate and grow in the regions with a high density of the coherent twin boundaries (CTBs). Very few voids were observed around HARGBs and LAGBs. Also, only a limited of number of small voids were observed in the vicinity of incoherent twin boundaries.

- Grain boundaries with low crystallographic order grain boundaries are efficient sinks for both vacancies and interstitials owing to the excess free volume, and thus do not stimulate biased point defect diffusion that promote void formation, which explains the observed lack of voids in the vicinity of HARGBs. 
- It is speculated that the observed high density of large voids in regions with finely spaced CTBs arises from the biased diffusion of point defects. Interstitials being more mobile and able to diffuse through CTBs can rapidly migrate and annihilate at free surfaces or other sinks such HARGBs. This leaves an excess of vacancies in the vicinity of the CTBs, which can coalesce and cause the nucleation of voids.

Given these observations, a nanocrystalline material having a high fraction of CTBs, though being resistant to radiation induced coarsening, may not possess the ideal topology for radiation resilience and may be prone to increased void formation and swelling. Hence a high interfacial area microstructures with an ideal mix of CSL and high-angle boundaries distributed in a fractal topology akin to microstructures of grain boundary engineered materials ([34]) may be more suitable for radiation tolerance. In such materials the topology of CTBs is quite different and may not promote such mechanisms for void formation, yet the topological connectivity in the grain boundary network may still provide stability against radiation induced coarsening

\section{Acknowledgements:}

This work was performed under the auspices of the U.S. Department of Energy by Lawrence Livermore National Laboratory under Contract DE-AC52-07NA27344. The efforts of TL and MK were supported by the U.S. Department of Energy (DOE), Office of Basic Energy Sciences, Division of Materials Science and Engineering under FWP\# SCW0939. In-situ electron irradiation studies were performed at the Research Center for Ultra-High Voltage Electron Microscopy in Osaka University. 


\section{References:}

[1] F.A. Nichols, Y.Y. Liu. Biased diffusion of radiation-produced point defects to interactive sinks, Journal of Nuclear Materials 108-109 (1982) 252-261.

[2] S.L. Dudarev, A.A. Semenov, C.H. Woo. Heterogeneous void swelling near grain boundaries in irradiated materials, Physical Review B 67 (2003).

[3] T. Leffers, B.N. Singh, S.N. Buckley, S.A. Manthorpe. VOID-SWELLING IN COLD-WORKED COPPER DURING HVEM IRRADIATION, Journal of Nuclear Materials 118 (1983) 60-67.

[4] T. Leffers, B.N. Singh, A.V. Volobuyev, V.V. Gann. EFFECTS OF HETEROGENEOUS SINK DISTRIBUTION ON VOID SWELLING, Philosophical Magazine a-Physics of Condensed Matter Structure Defects and Mechanical Properties 53 (1986) 243-257.

[5] D.I.R. Norris. Use of High Voltage Electron Microscope to Simulate Fast Neutron-Induced Void Swelling in Metals, Journal of Nuclear Materials 40 (1971) 66.

[6] B.N. Singh, A. Horsewell, D.S. Gelles, F.A. Garner. VOID SWELLING IN COPPER AND COPPERALLOYS IRRADIATED WITH FISSION NEUTRONS, Journal of Nuclear Materials 191 (1992) 1172-1176.

[7] M.J. Demkowicz, R.G. Hoagland, B.P. Uberuaga, A. Misra. Influence of interface sink strength on the reduction of radiation-induced defect concentrations and fluxes in materials with large interface area per unit volume, Physical Review B 84 (2011) 104102.

[8] X.-M. Bai, A.F. Voter, R.G. Hoagland, M. Nastasi, B.P. Uberuaga. Efficient Annealing of Radiation Damage Near Grain Boundaries via Interstitial Emission, Science 327 (2010) 1631-1634.

[9] B.N. Singh. Influence of Grain-Boundaries on Void Growth, Philosophical Magazine 28 (1973) 1409-1413.

[10] B.N. Singh. Void Volume Swelling Dependent on Grain-size in an Austenitic Stainless-Steel, Nature-Physical Science 244 (1973) 142-142.

[11] B.N. Singh. Effect of Grain-size on Void Formation during High-energy Electron-irradiation of Austenitic Stainless-steel, Philosophical Magazine 29 (1974) 25-42.

[12] B.N. Singh, A.J.E. Foreman. Calculated Grain Size-dependent Vacancy Supersaturation and its effect on void formation, Philosophical Magazine 29 (1974) 847-857.

[13] T. LaGrange, B.W. Reed, M. Wall, J. Mason, T.W. Barbee, M. Kumar. Topological view of the thermal stability of nanotwinned copper, Applied Physics Letters 102 (2013) 011905.

[14] L. Lu, M.L. Sui, K. Lu. Superplastic extensibility of nanocrystalline copper at room temperature, Science 287 (2000) 1463-1466.

[15] O. Anderoglu, A. Misra, H. Wang, X. Zhang. Thermal stability of sputtered Cu films with nanoscale growth twins, Journal of Applied Physics 103 (2008).

[16] X. Zhang, O. Anderoglu, R.G. Hoagland, A. Misra. Nanoscale growth twins in sputtered metal films, JOM 60 (2008) 75-78.

[17] M. Kumar, C.A. Schuh. Preface to the viewpoint set on grain boundary engineering, Scripta Materialia 54 (2006) 961-962.

[18] A.M. Hodge, Y.M. Wang, T.W. Barbee. Large-scale production of nano-twinned, ultrafine-grained copper, Materials Science and Engineering a-Structural Materials Properties Microstructure and Processing 429 (2006) 272-276.

[19] K. Niwase, F. Phillipp, W. Sigle, A. Seeger. Void formation close to stacking fault tetrahedra in heavily electron irradiated pure Ag and Cu, Journal of Nuclear Materials 271 (1999) 261-265.

[20] E.F. Rauch, M. Veron, J. Portillo, D. Bultreys, Y. Maniette, Nicolopoulos. Automatic Cyrstal Orientation and Phase Mapping in TEM by Precession Diffraction, Microscopy and Analysis 22 (2008) S5S8.

[21] W. Bollmann. CLASSIFICATION OF CRYSTALLINE INTERFACES BY MEANS OF O-LATTICE METHOD, Journal of Microscopy-Oxford 102 (1974) 233-239. 
[22] D.I.R. Norris. Voids in irradiated metals . I, Radiation Effects 14 (1972) 1-37.

[23] C. Campana, K.P. Boyle, R.E. Miller. Grain boundary motion assisted via radiation cascades in bcc Fe, Physical Review B 78 (2008).

[24] K. Nakata, Y. Katano, I. Masaoka, K. Shiraishi. GRAIN-BOUNDARY MIGRATION DURING ELECTRON-IRRADIATION IN AUSTENITIC STAINLESS-STEELS, Journal of Nuclear Materials 133 (1985) 575579.

[25] N. Sakaguchi, T. Shibayama, H. Kinoshita, H. Takahashi. Atomistic observation of radiationinduced grain-boundary movement in Fe-Cr-Ni alloy under electron irradiation, Philosophical Magazine Letters 81 (2001) 691-696.

[26] K.Y. Yu, D. Bufford, F. Khatkhatay, H. Wang, M.A. Kirk, X. Zhang. In situ studies of irradiationinduced twin boundary migration in nanotwinned Ag, Scripta Materialia 69 (2013) 385-388.

[27] K.Y. Yu, D. Bufford, C. Sun, Y. Liu, H. Wang, M.A. Kirk, M. Li, X. Zhang. Removal of stacking-fault tetrahedra by twin boundaries in nanotwinned metals, Nature Communications 4 (2013).

[28] S.F. Li, J.F. Lind, M. Kumar. Evolution of experimentally derived grain boundary energy distribution in polycrystalline microstructures during thermal coarsening. Submitted to Physical Review Letters, 2014.

[29] R.W. Grimes, R.J.M. Konings, L. Edwards. Greater Tolerance for Nuclear Materials, Nature 7 (2008) 683-685.

[30] W.Z. Han, M.J. Demkowicz, E.G. Fu, Y.Q. Wang, A. Misra. Effect of grain boundary character on sink efficiency, Acta Materialia 60 (2012) 6341-6351.

[31] M.I. Mendelev, A.H. King. The interactions of self-interstitials with twin boundaries, Philosophical Magazine 93 (2013) 1268-1278.

[32] V. Bulatov, B.W. Reed, M. Kumar. Grain boundary energy function for FCC metals, Acta Materialia 65 (2014) 61.

[33] M.J. Demkowicz, O. Anderoglu, X. Zhang, A. Misra. The influence of Sigma 3 twin boundaries on the formation of radiation-induced defect clusters in nanotwinned $\mathrm{Cu}$, Journal of Materials Research 26 (2011) 1666-1675.

[34] B.W. Reed, M. Kumar, R.W. Minich, R.E. Rudd. Fracture roughness scaling and its correlation with grain boundary network structure, Acta Materialia 56 (2008) 3278-3289. 
Figures:
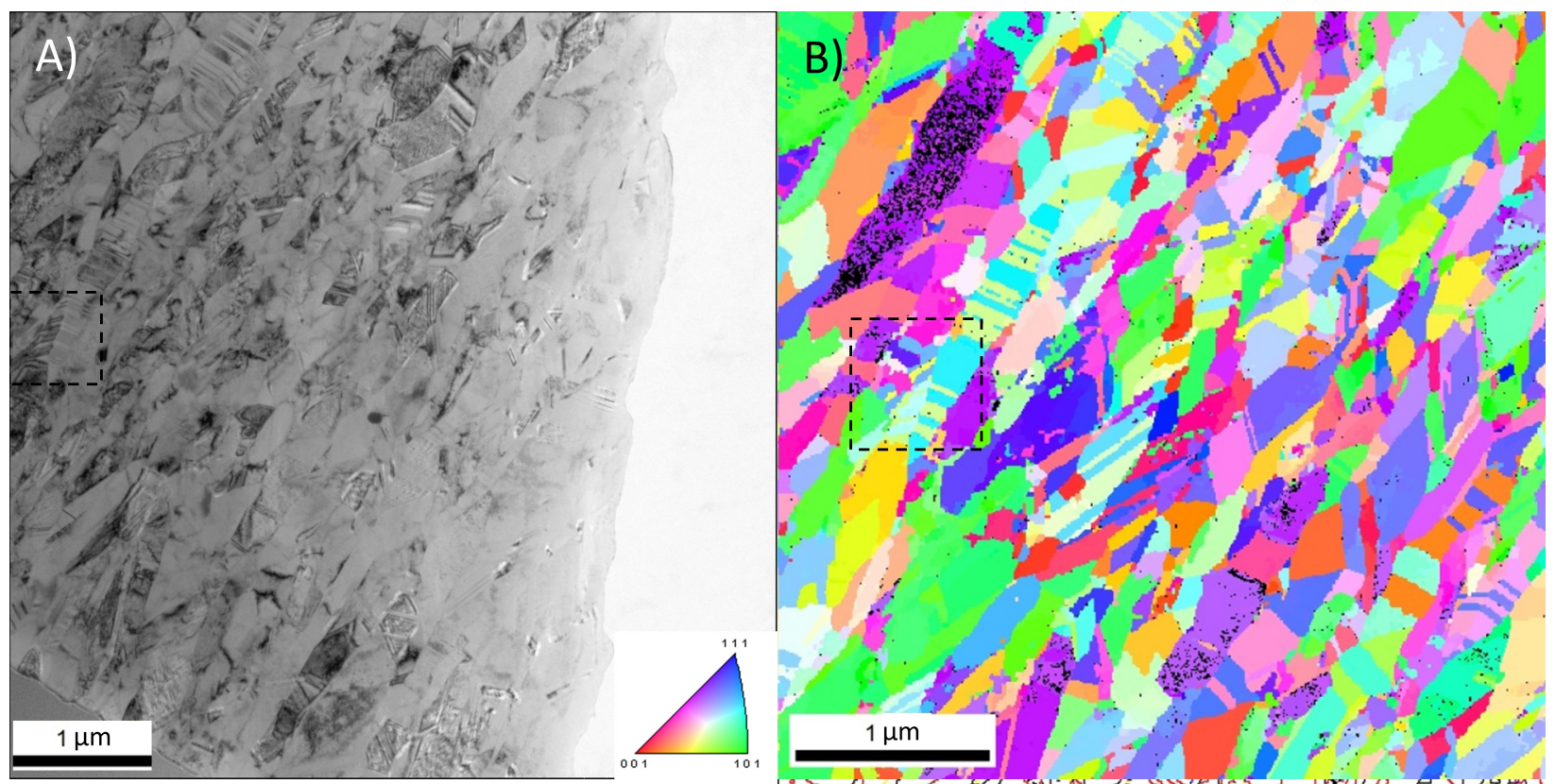

C)
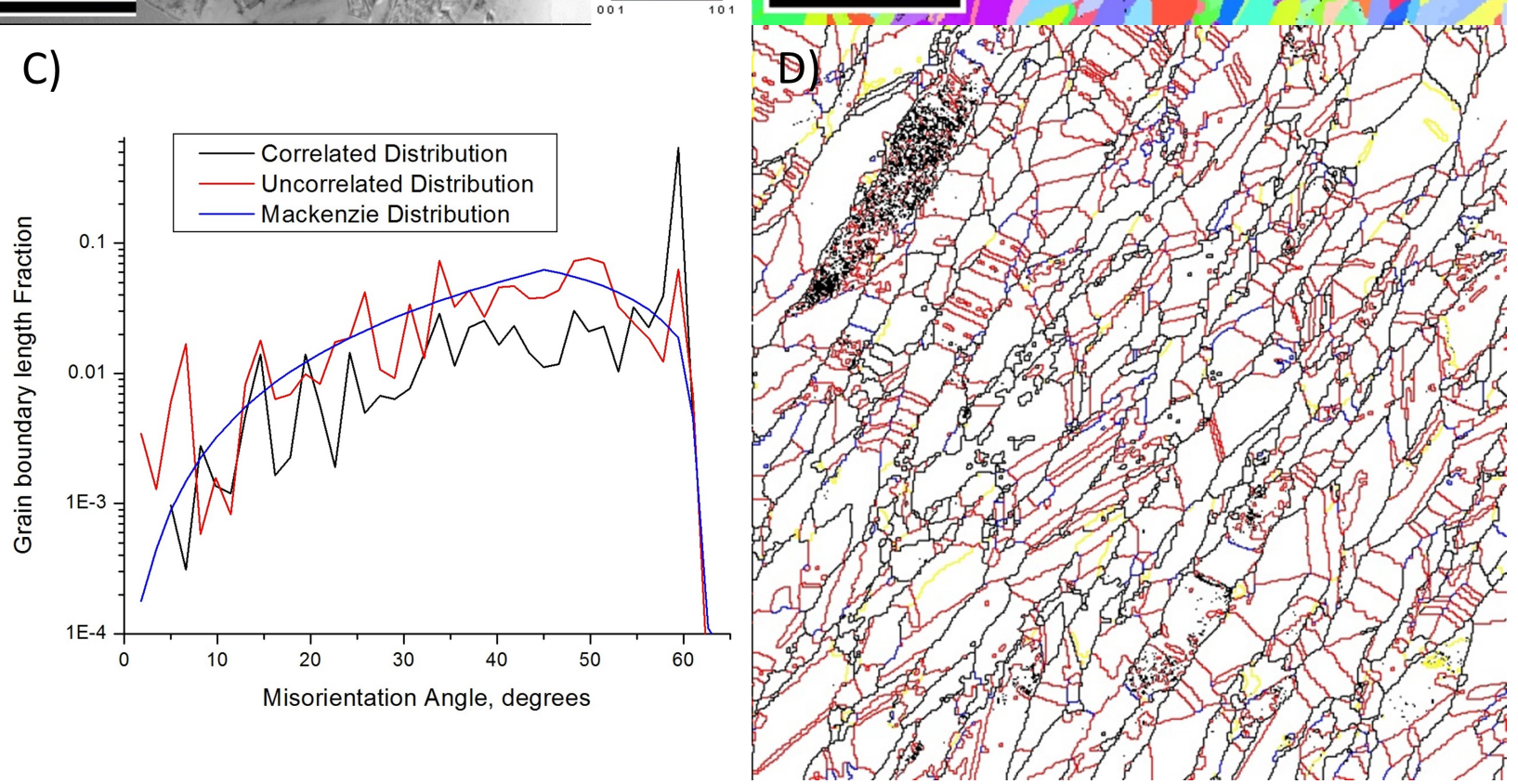

Figure 1. Crystallographic analysis of the as-deposited nt-Cu films: A) Bright-field image of crosssectional TEM sample of nt-Cu foil prior to electron irradiation; B) Corresponding inverse pole figure crystallographic orientation map with $<100>$ grain normal out of plane; C) Measured misorientation angle distribution plot emphasizing the contrast between the measured nt-Cu and the MacKenzie (random texture) distribution; D) Grain boundary character map of the area in A) and $B$ ), which also highlights the columnar nature of the grain structure in these films. High angle boundaries (misorientation $>15^{\circ}$ ) are colored black, low angle boundaries $\left(<15^{\circ}\right.$ ) are colored 
yellow, $\Sigma 3$ CSL boundaries are red and $\Sigma 9$ CSL boundaries are colored blue. The region highlighted by the dashed-line box in A) and B) indicates the area of irradiation experiments shown in Fig. 2 . 

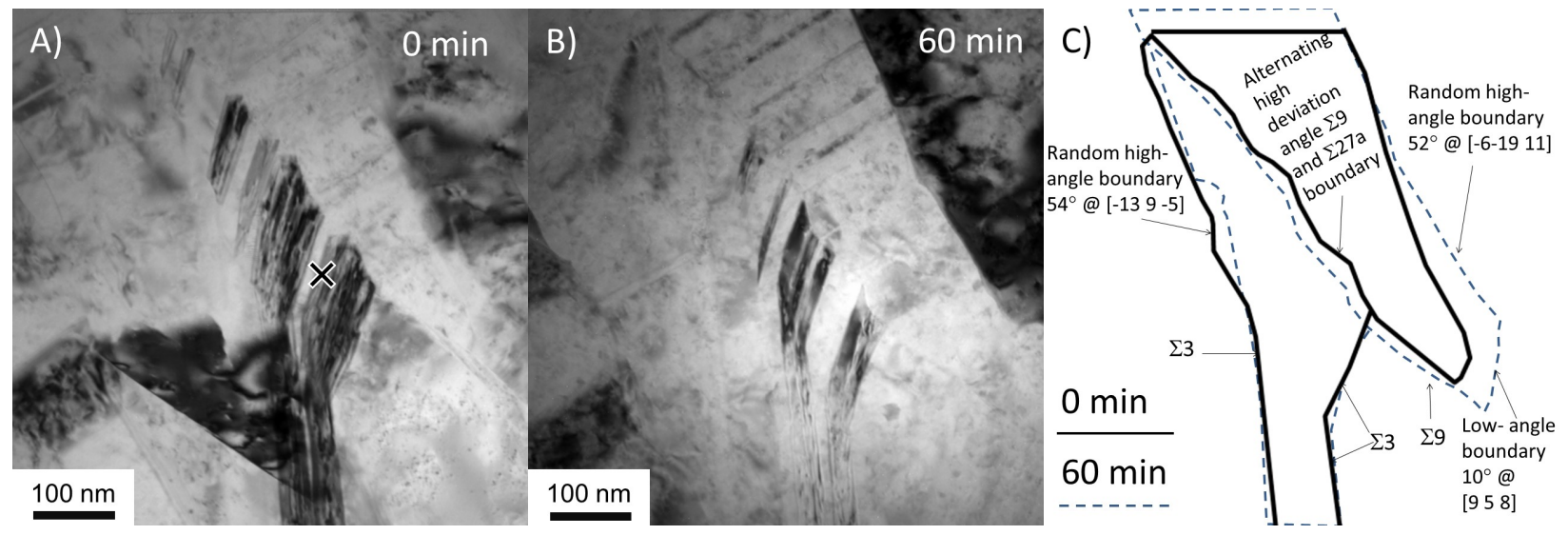

Figure 2. Ambient temperature electron irradiation experiments: A) Bright-field cross-sectional TEM image of the irradiated zone prior to electron irradiation. The cross marks the center of the electron beam; B) Bright-field TEM image of the irradiated zone after 60 mins of irradiation to a dose of $8.7 \times 10^{27} \mathrm{e}^{-} \mathrm{m}^{-2}$; C) Traces of the position of selected grain boundaries before and after irradiation. Solid black lines denote the position of the grain boundaries before irradiation and dashed blue lines are after irradiation. Boundaries are characterized by the misorientation angle and rotation axis. Significant migration of the $52^{\circ}$ boundary was observed while positions of the CSL boundaries were virtually unchanged. 


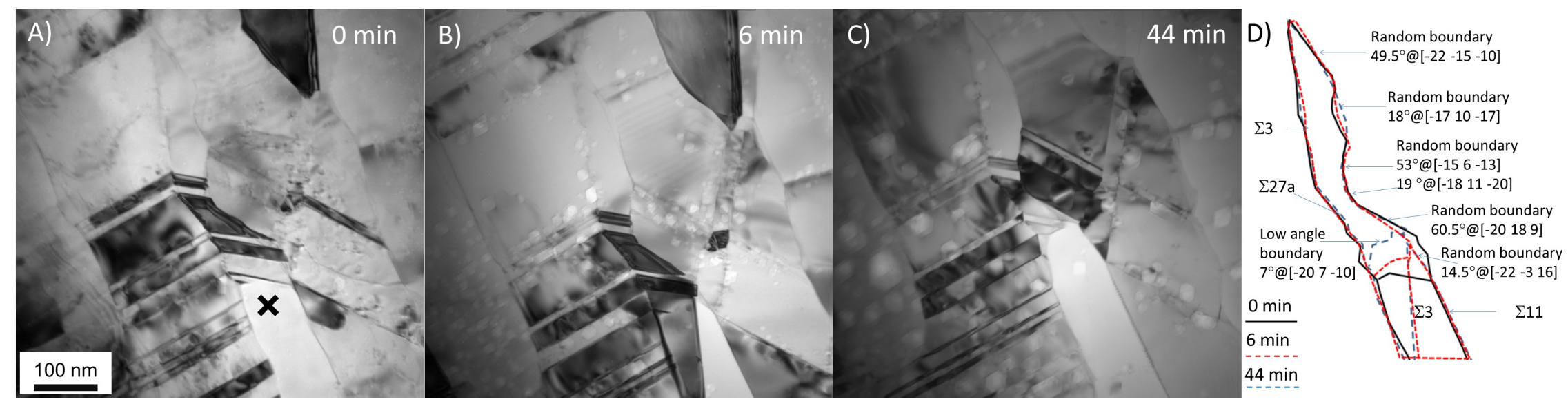

Figure 3. Electron irradiation experiments at 573K: A) Bright-field cross-sectional TEM image of the irradiated zone prior to electron irradiation. The cross marks the center of the electron beam; B) Bright-field TEM image of the irradiated zone after 6 mins of irradiation to a dose of $8.7 \times 10^{26} \mathrm{e}^{-} \mathrm{m}^{-2}$. Void formation is observed in the vicinity of the CTBs; C) Bright-field TEM image after irradiating for 44 mins to a dose of $6.4 \times 10^{27} \mathrm{e}^{-} \mathrm{m}^{-2}$. The pre-existing voids grew and appear to coalesce and facet. D) Traces of the position of selected grain boundaries before and after irradiation. Solid black lines are before irradiation; dashed red and blue lines are after irradiating the sample for 6 and 44 mins, respectively. Boundaries are characterized by the misorientation angle and rotation axis. Significant migration of the $7^{\circ}$ boundary was observed while again position of the CSL boundaries was unchanged. 


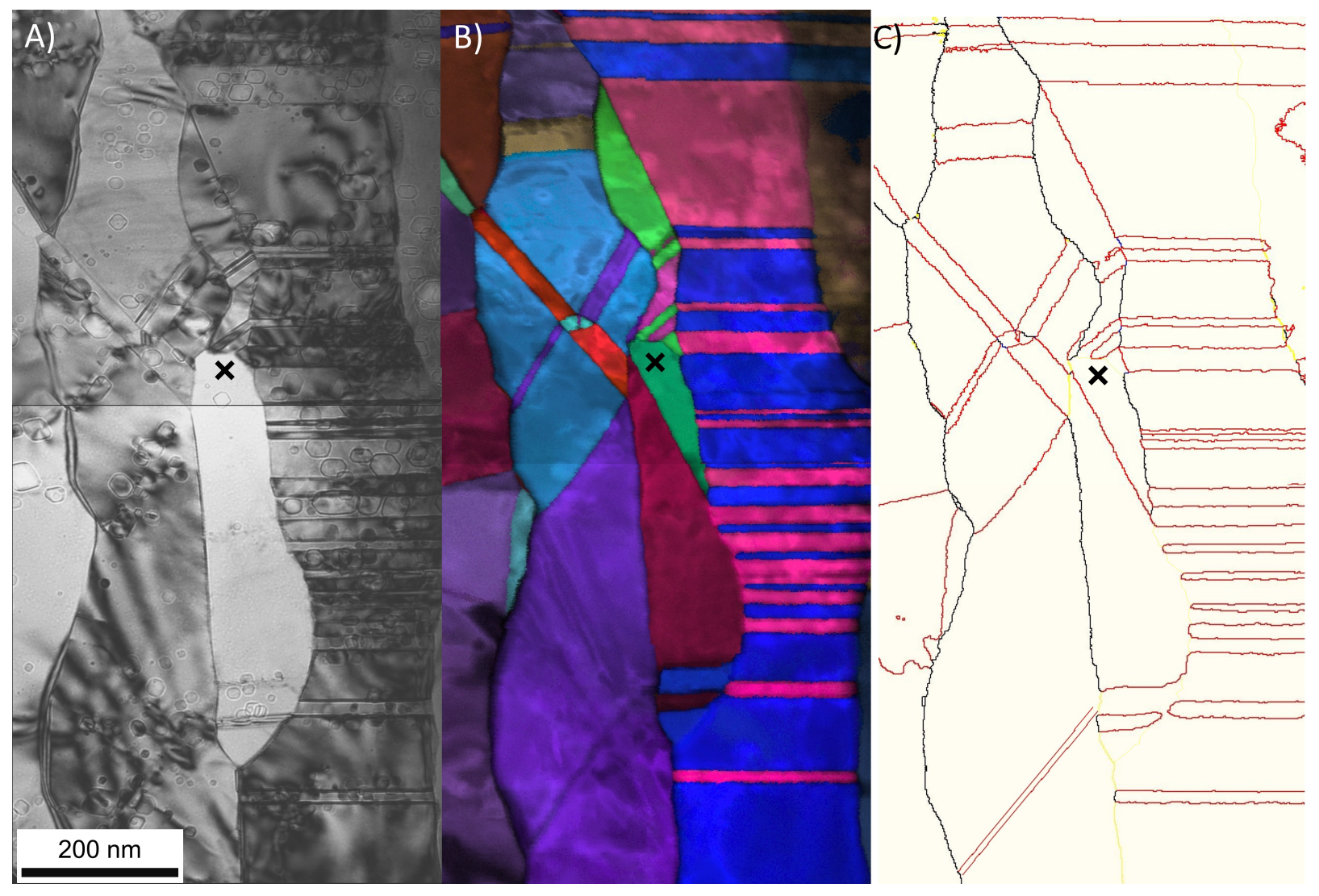

Figure 4. Crystallographic analysis of the region on $\mathrm{nt}-\mathrm{Cu}$ foil irradiated at $573 \mathrm{~K}, \mathrm{~A}$ ) Bright-field cross-sectional TEM image of nt- $\mathrm{Cu}$. The cross marks the center of the interaction area with the electron beam, as shown in Fig. 3; B) corresponding (001) inverse pole figure crystallographic orientation map. C) Grain boundary character map, High-angle boundaries with a misorientation $>15^{\circ}$ are colored black, low-angle boundaries $<15^{\circ}$ are colored yellow, $\Sigma 3$ CSL boundaries are red and $\Sigma 9$ CSL boundaries are colored blue. Large voids tend to be in the vicinity of $\Sigma 3$ CSL boundaries and absent around high-angle and low-angle boundaries. 